\title{
The role and significance of magnetic resonance imaging and miRNA-125b expression level in diagnosis of head and neck squamous cell carcinoma
}

\author{
Qibiao Huang ${ }^{1}$, Xiao Zhang², Biao Gong 3 , Dongquan $\mathrm{Ge}^{4}$ \\ ${ }^{1}$ CT Room, Shanxian Central Hospital, Heze, Shandong, China \\ 2Department of Radiology, Binzhou People’s Hospital, Bincheng District, Binzhou, Shandong, China \\ ${ }^{3}$ Imaging Medicine and Nuclear Medicine, Shandong University School of Medicine, Lixia District, Jinan, Shandong, China
}

${ }^{4}$ Department of Medical Imaging, Pingdu People's Hospital, Pingdu, Shandong, China

Adv Dermatol Allergol 2022; XXXIX (1): 110-115

DOI: https://doi.org/10.5114/ada.2021.104701

\begin{abstract}
Introduction: Diagnosis of head and neck squamous cell carcinoma (SCC) is an important challenge for clinicians, and finding new approaches to better diagnosis is the basis of a new area of cancer research.

Aim: To investigate the diagnostic value and significance of MRI combined with miRNA-125 expression in head and neck SCC.

Material and methods: Sixty patients with head and neck SCC were selected as the tumour group, and 20 healthy volunteers as the control group. All subjects were examined by magnetic resonance imaging (MRI) perfusion imaging. Peripheral venous blood was collected from all patients and healthy volunteers. The expression level of miRNA-125 in serum was detected by RT-qPCR, and the levels of interleukin-2 (IL-2), tumor necrosis factor- $\alpha$ (TNF- $\alpha$ ), interferon- $\gamma$ (IFN- $\gamma$ ), and transforming growth factor $\beta 1$ (TGF- $\beta 1$ ) in serum were detected. Meanwhile, tumour tissues and adjacent non-tumour tissues of patients were collected to detect the mRNA expression level of ERBB2. Results: Compared with the control group, the expression level of miRNA-125b in serum of patients decreased with a statistically significant difference $(p<0.05)$. Compared with adjacent non-tumour tissues, the expression level of ERBB2 mRNA in tumour tissues of patients was significantly increased, with a statistically significant difference $(p<0.05)$. MiRNA-125b in serum was negatively correlated with tumour size and number of metastatic foci $(p<0.05)$. The results of enzyme-linked immunosorbent assay showed that the levels of IL-2, TNF- $\alpha$, and TGF- $\beta 1$ in the patient's serum increased while the levels of IFN- $\gamma$ decreased significantly, with a statistically significant difference $(p<0.05)$.
\end{abstract}

Conclusions: Detection of miRNA-125b expression level is complementary to MRI diagnosis of head and neck SCC.

Key words: magnetic resonance imaging , miRNA-125b, squamous cell carcinoma.

\section{Introduction}

Tumours in the neck, ear-nose-throat, as well as oral and maxillofacial regions are collectively referred to as head and neck cancers, such as tongue squamous cell carcinoma (SCC), laryngeal SCC, nasal sinus carcinoma, gingival carcinoma, and thyroid carcinoma. Some investigations showed that the proportion of head and neck cancer in human tumours is about 2-4\% [1]. About 30\% to $50 \%$ of patients with head and neck cancer will relapse or metastasise after surgical treatment [2]. Currently, there are various clinical diagnostic methods for head and neck cancer, including computed tomography
(CT), CT perfusion, magnetic resonance imnaging (MRI), MRI diffusion-weighted imaging, MRI perfusion imaging, etc. These diagnostic methods have their own advantages and disadvantages, and are selected according to the situation in clinical practice [3]. In recent years, due to the development of medical molecular biology, detection of microRNA expression in peripheral blood is favoured for its advantages of low trauma and high sensitivity, playing an increasingly irreplaceable role in the diagnosis of tumours. MicroRNAs comprise a kind of endogenous non-coding RNA molecules that regulate the expression of protein-coding genes at post-transcriptional level [4].

\footnotetext{
Address for correspondence: Dongquan Ge, Department of Medical Imaging, Pingdu People's Hospital, 112 Yangzhou Road, Pingdu, Shandong Province, 266700, China, e-mail: dongquange@yahoo.com Received: 22.10.2020, accepted: 9.11.2020. 
Most microRNAs are closely related to cell proliferation, differentiation, and apoptosis, and many of them are found to be potential markers for tumour diagnosis and prognosis [5-7]. The MiRNA-125 family comprises highly conserved non-coding small RNA molecules in evolution, including two subfamilies of miRNA-125a and miRNA$125 \mathrm{~b}$, whose abnormal expression is closely associated with tumour occurrence and development $[8,9]$. Thereinto, miRNA-125b is significantly differentially expressed in various malignant tumours and has become a classic marker for cancer diagnosis, treatment, and prognosis [10-13]. Its downstream target genes include p53, Bak1, and cytokines IL- 6 and TGF- $\beta[9,14]$. Moreover, many studies have shown that miRNA-125b can inhibit cancer development and exert a protective effect on patients [12, 15-18]. For example, studies by Shang et al. showed that miRNA-125b can target proto-oncogene ERBB2, and the level of miRNA-125b in cancer tissues of endometrial cancer patients decreases, resulting in enhanced cancer invasion capability [16]. The ERBB2 gene encodes CerbB-2 protein, also known as Her 2, is one of the members of epidermal growth factor receptor family (EGFR), and is the most thoroughly studied oncogene in breast cancer. Research showed that over $30 \%$ of human malignant tumour tissues, such as ovarian cancer, cervical cancer, prostate cancer, and head and neck squamous cell carcinoma, are accompanied by C-erbB-2 gene amplification and overexpression of 185 protein product $[19,20]$.

\section{Aim}

The purpose is to explore the diagnostic value and significance of MRI combined with miRNA-125 expression level in head and neck SCC.

\section{Material and methods \\ Clinical data}

A total of 60 patients with head and neck SCC diagnosed and treated in Shanxian Central Hospital from January 2014 to February 2019 were selected as the tumour group. Among them, there were 36 males and 24 females, aged from 36 to 68 years. There were 9 cases of tongue SCC, 10 cases of laryngeal squamous cell carcinoma, 13 cases of sinus carcinoma, 10 cases of gingival carcinoma, and 18 cases of thyroid carcinoma. Meanwhile, a total of 20 healthy volunteers with no abnormalities in head MRI angiography were selected as the control group. Serum was collected from all patients and subjects before MRI examination, and samples of tumour tissue and adjacent non-tumour tissue were taken from patients. All subjects were informed of the purpose of the examination and signed an informed consent form before the examination. The study was approved by the hospital Ethics Committee.

\section{Declarations}

Ethics approval and consent to participate: This study was approved by the Medical Ethics Committee of Shanxian Central Hospital

\section{Experimental methods \\ MRI examination}

Cranial MRI was performed on all patients and healthy subjects using general MRI to determine the location of tumour in the patients and to exclude disease in healthy subjects. Then the largest target area was selected for the patient, $20 \mathrm{ml}$ of contrast agent was injected from elbow vein using a high-pressure syringe (Medrad Stellant), and the pipeline was flushed with $20 \mathrm{ml}$ of normal saline immediately after the injection. The layer thickness was set at $5 \mathrm{~mm}$, interlayer clearance was $1.5 \mathrm{~mm}$, TE54 ms, and the pixel matrix was $128 \times 128$. MRI perfusion imaging was performed and 40 T2-weighted measurements were obtained at 2-s intervals to observe whether there was metastasis of the tumour. This inspection used a Siemens 1.5T 18-channel MRI system for scanning inspection.

\section{Total RNA extraction and real-time quantitative $P C R(q R T-P C R)$}

Serum miRNA and tissue mRNA were extracted by TRIzol method. For the detection of serum hsa-miR$125 \mathrm{~b}$, reverse transcription was performed using a stem loop method. Quantitative PCR was conducted using an SYBR Green qPCR SuperMix kit (Invitrogen). The primer sequence was as follows: hsa-miR-125b: F: 5'-CACCTGTATGTGCGTGATTGTATATG-3', R: 5'-CCTCAGGCATCAATAACATGCA-3'; U6: F:5' - CTCGCTTCGGCAGCACA-3', R: 5'AACGCTTCACGAATTTGCGT-3'.

For tissue ERBB2 mRNA detection, cDNA is first synthesised using a reverse transcription kit (Cat \#: $6210 \mathrm{~A}$, TaKaRa). The synthesised cDNA can be directly used for fluorescence quantitative PCR or stored at $-20^{\circ} \mathrm{C}$. Realtime fluorescence quantitative PCR was performed using an SYBR Premix Ex Taq ${ }^{\text {TM }}$ II kit (purchased from the TAKARA company), and PCR amplification was performed using ABI PRISM ${ }^{\oplus} 7500$ Sequence Detection System. The primer sequence was as follows: ERBB2: F: 5'-CACATGCAAAGCTACTC-3', R: 5'-GGTGCACACTCACTTT-3'; 18srRNA: F: 5'-CCTGGATACCGCAGCTAGGA-3', R: 5'- GCGGCGCAATACGAATGCCCC-3'.

\section{Enzyme-linked immunosorbent assay (ELISA)}

Using commercial kits, the levels of IL-2 (Cat\#: EHC003, Neobioscience), TNF- $\alpha$ (Cat\#: EHC103a, Neobioscience), IFN- $\gamma$ (Cat\#: EHC102g, Neobioscience), and TGF- $\beta 1$ (Cat\#: EHC107b, Neobioscience) in patients' serum were detected in accordance with manufacturer's instructions. 


\section{Statistical analysis}

All data were processed by SPSS 21.0 statistical software (USA), and the measurement data were expressed as mean \pm standard deviation (SD). The comparison between the two groups was performed by t-test, and the comparison between multiple groups was conducted by one-way ANOVA. $P<0.05$ indicated that the difference was statistically significant.

\section{Results}

MRI examination to determine the patient's tumour

First of all, we performed cranial MRI examination on all patients with head and neck cancer and healthy subjects to determine the tumour location and size among the patients (Figure 1). MRI examination results of healthy volunteers were negative. MRI examination further confirmed the metastasis conditions of tumour patients.

\section{Cytokine test results.}

The serum cytokine expression levels of all subjects were detected by ELISA. As a result, the levels of IL-2, TNF- $\alpha$, and TGF- $\beta 1$ in the patient's serum increased significantly (Figures $2 \mathrm{~A}-\mathrm{C}$ ), while the levels of IFN- $\gamma$ were significantly decreased (Figure $2 \mathrm{D}$ ), with statistically significant differences $(p<0.05)$. The above cytokine detection results were basically consistent with MRI detection results.

The expression level of miRNA-125b in serum of patients with head and neck cancer decreased

In order to explore whether the serum miRNA-125b level is correlated with the metastasis of head and neck cancer, q-PCR was used to detect the expression of miR$\mathrm{NA}-125 \mathrm{~b}$ in the serum of patients and healthy subjects. The results are shown in Figure 3. Compared with the control group, the serum miRNA-125b level of patients is significantly reduced $(p<0.05)$, i.e. the serum miRNA$125 \mathrm{~b}$ level is negatively correlated with tumour malignancy.

\section{Comparison of ERBB2 gene expression levels in tumour tissues and adjacent tumour or non-tumour tissues}

It has been reported that ERBB2 is the target gene of miRNA-125b, so we further used q-PCR to detect the expression of ERBB2 gene in tumour tissues and adjacent non-tumour tissues of all patients with head and neck cancers. The results (Figure 4) showed that the mRNA expression level of ERBB2 in tumour tissues was significantly higher than that in adjacent para-tumour tissues $(p<0.05)$.

\section{Discussion}

In recent years, MRI perfusion imaging, which is widely used in the diagnosis and follow-up of head and neck cancer and other malignant tumours, is a functional imaging examination method that can well reflect tumour haemodynamics. With the advantages of fast imaging and no radiation, it can scan the whole brain perfusion at one time in head scanning and is not limited to the selected level. MRI perfusion imaging plays a significant part in tumour diagnosis and differentiation [21, 22] and evaluation of tumour staging $[23,24]$. However, MRI perfusion imaging still has some deficiencies in clinical application due to the non-linear relationship between
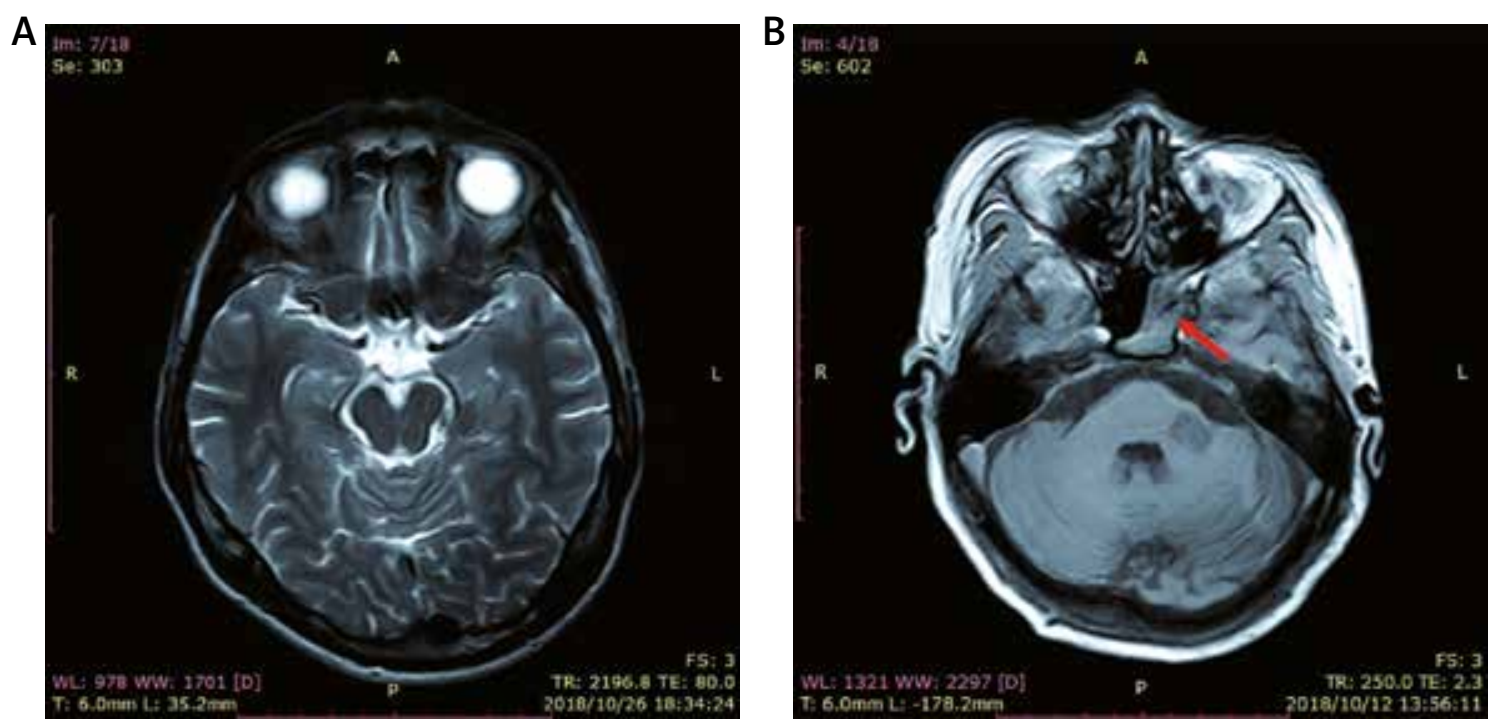

Figure 1. MRI images of the control group (A) and the tumour group (B) 

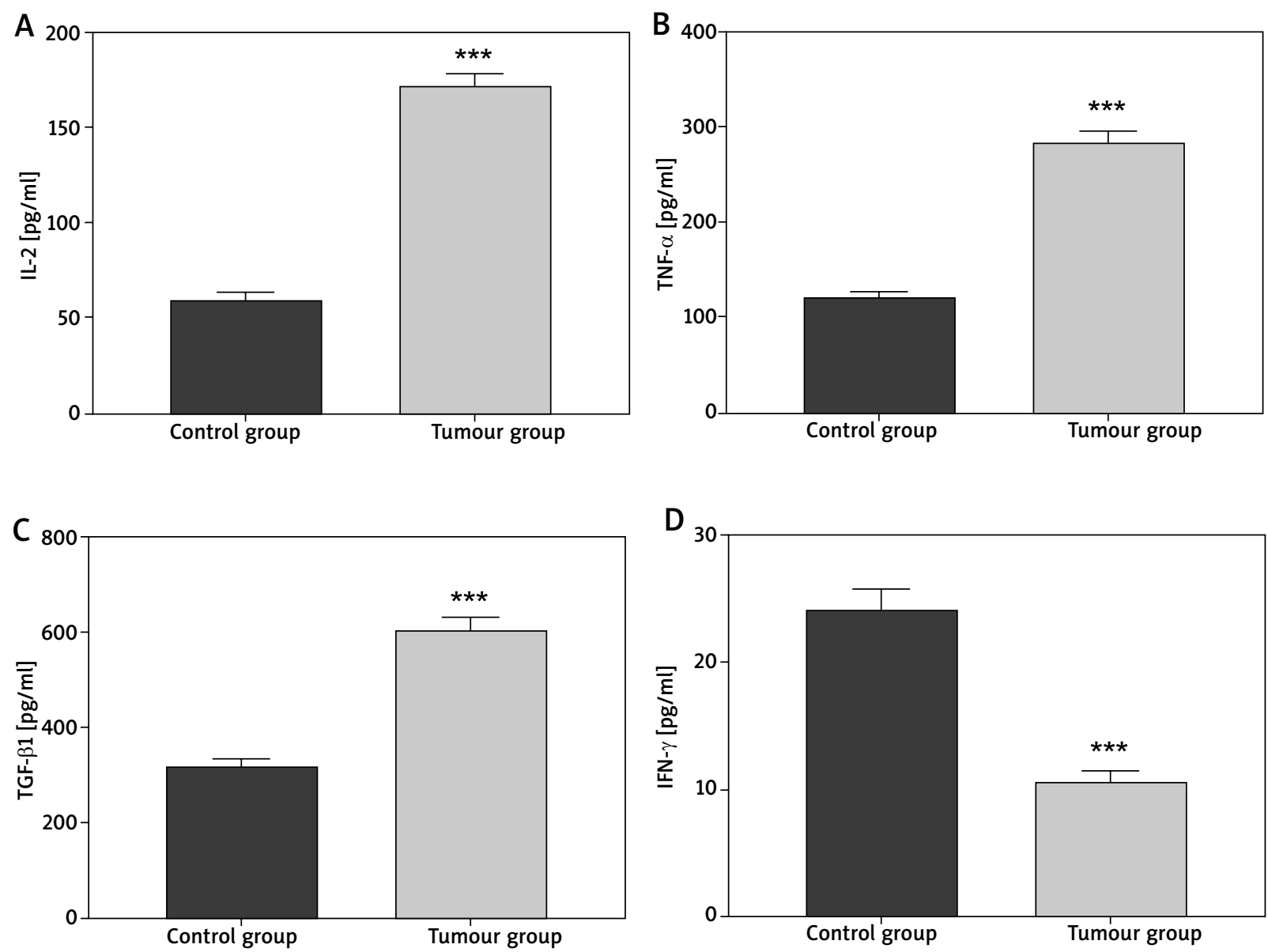

Figure 2. Expression levels of IL-2 (A), TNF- $\alpha$ (B), TGF- $\beta 1$ (C), and IFN- $\gamma$ (D) in serum of the control group and the tumour group. ${ }^{* * *} P<0.001$ vs. control group

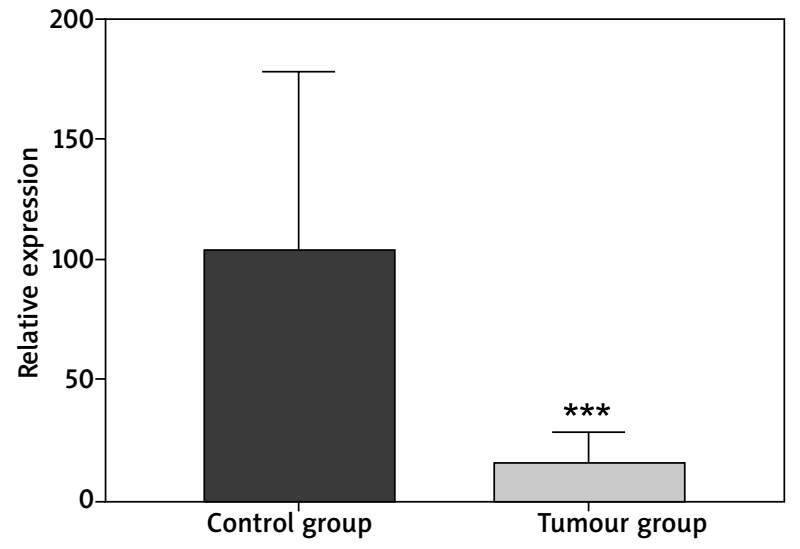

Figure 3. The expression level of miRNA-125b in serum of the control group and the tumour group. ${ }^{\star *} P<0.001 \mathrm{vs.}$ control group

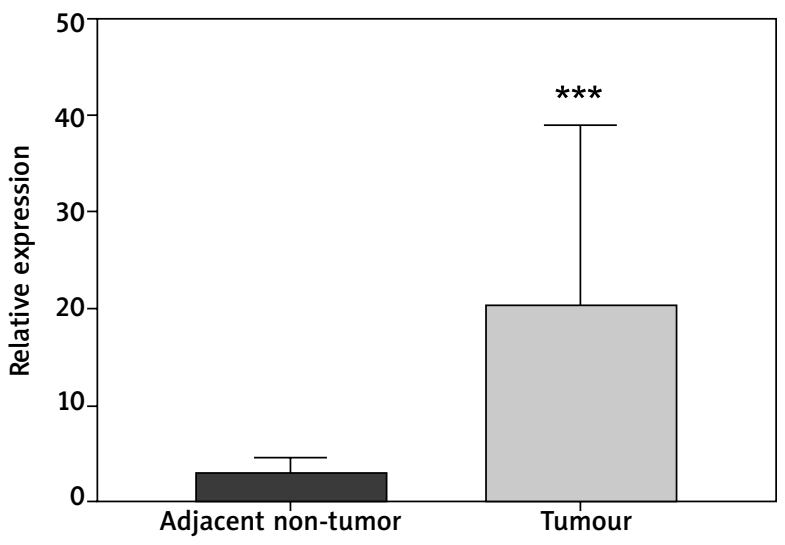

Figure 4. ERBB2 gene expression levels in tumour tissues and adjacent non-tumour tissues. ${ }^{* *} P<0.001$ vs. adjacent non-tumour tissues group 
tissue signal intensity and contrast agent concentration, which affects quantitative measurement.

MicroRNAs, as a non-coding regulatory single-stranded small molecule RNA with a total length of 19-25 ribonucleotides, is generated by shearing a single-stranded RNA precursor with a hairpin ring structure. Through complementary pairing with the 3'- end non-coding region (3-UTR) of the target mRNA molecule, microRNA inhibits the translation of the target mRNA and achieve post-transcriptional expression regulation [25]. A large number of studies have shown that microRNA is closely related to the occurrence and development of tumours. Meanwhile, some research in recent years has found that microRNA in peripheral venous blood has a stable physicochemical state and properties, and its expression in tumour patients is significantly different from that in the normal human body. Many researches have indicated the value of miRNA as a specific tumour marker [26]. Among them, miRNA-125b can play a critical role in promoting cancer and inhibiting cancer in malignant tumours of different tissues $[27,28]$. In this study, the expression level of miRNA-125b in the serum of patients with head and neck cancer showed a downward trend compared with that of healthy patients, and correspondingly, the expression level of its target gene ERBB2 in tumour tissue decreased.

In this study, combining MRI examination with mRNA expression detection, it was found that the expression level of related mRNA in serum of patients with head and neck cancer was lower than that of normal people. The difference in level was statistically significant and negatively correlated with the tumour size in the imaging diagnosis results.

\section{Conclusions}

As a result of the study, we believe that the combination of the two diagnostic and examination methods in clinical practice can adopt good points and avoid shortcomings to maximise their respective advantages, and improve the diagnostic efficiency and accuracy of head and neck cancer recurrence.

\section{Conflict of interest}

The authors declare no conflict of interest.

\section{References}

1. Marur S, Forastiere AA. Head and neck cancer: changing epidemiology, diagnosis, and treatment. Mayo Clin Proc 2008; 83: 489-501.

2. Gupta T, Master Z, Kannan S, et al. Diagnostic performance of post-treatment FDG PET or FDG PET/CT imaging in head and neck cancer: a systematic review and meta-analysis. Eur J Nucl Med Mol Imaging 2011; 38: 2083-95.
3. Chen ST, Qi L, Zhang XJ, et al. An evaluation of the technologies of medical imaging in the diagnosis of head and neck tumor. Health Research 2012; 32: 302-5.

4. Ambros V. The functions of animal microRNAs. Nature 2004; 431: 350-5.

5. Kent OA, Mendell JT. A small piece in the cancer puzzle: microRNAs as tumor suppressors and oncogenes. Oncogene 2006; 25: 6188-96.

6. Calin GA, Croce CM. MicroRNA signatures in human cancers. Nat Rev Cancer 2006; 6: 857-66.

7. Cummins JM, Velculescu VE. Implications of micro-RNA profiling for cancer diagnosis. Oncogene 2006; 25: 6220-7.

8. Masood N, Basharat Z, Khan T, Yasmin A. Entangling relation of micro RNA-let7, miRNA-200 and miRNA-125 with various cancers. Pathol Oncol Res 2017; 23: 707-15.

9. Yin H, Sun Y, Wang X, et al. Progress on the relationship between miR-125 family and tumorigenesis. Exp Cell Res 2015; 339: 252-60.

10. Li J, Fang L, Yu W, Wang Y. MicroRNA-125b suppresses the migration and invasion of hepatocellular carcinoma cells by targeting transcriptional coactivator with PDZ-binding motif. Oncol Letters 2015; 9: 1971-5.

11. Zhang X, Chu X, Gong X, et al. The expression of miR-125b in Nrf2-silenced A549 cells exposed to hyperoxia and its relationship with apoptosis. J Cell Mol Med 2020; 24: 965-72.

12. Zhao X, He W, Li J, et al. MiRNA-125b inhibits proliferation and migration by targeting SphK1 in bladder cancer. Am J Transl Res 2015; 7: 2346-54.

13. Zhou HC, Fang JH, Shang LR, et al. MicroRNAs miR-125b and miR-100 suppress metastasis of hepatocellular carcinoma by disrupting the formation of vessels that encapsulate tumour clusters. J Pathol 2016; 240: 450-60.

14. Shi XB, Xue L, Yang J, et al. An androgen-regulated miRNA suppresses Bak1 expression and induces androgen-independent growth of prostate cancer cells. Proc Natl Acad Sci USA 2007; 104: 19983-8.

15. Cui F, Li X, Zhu X, et al. MiR-125b inhibits tumor growth and promotes apoptosis of cervical cancer cells by targeting phosphoinositide 3-kinase catalytic subunit delta. Cell Physiol Biochem 2012; 30: 1310-8.

16. Shang C, Lu YM, Meng LR. MicroRNA-125b down-regulation mediates endometrial cancer invasion by targeting ERBB2. Med Sci Monitor 2012; 18: Br149-55.

17. Brock M, Hottinger S, Diebold M, et al. Low tissue levels of miR-125b predict malignancy in solitary fibrous tumors of the pleura. Respir Res 2017; 18: 43.

18. Bu Q, You F, Pan G, et al. MiR-125b inhibits anaplastic thyroid cancer cell migration and invasion by targeting PIK3CD. Biomed Pharmacother 2017; 88: 443-8.

19. Gullick WJ. The role of the epidermal growth factor receptor and the c-erbB-2 protein in breast cancer. Int J Cancer Suppl 1990; 5: 55-61.

20. Hynes NE, Stern DF. The biology of erbB-2/neu/HER-2 and its role in cancer. Biochim Biophys Acta 1994; 1198: 165-84.

21. Bisdas S, Fetscher S, Feller AC, et al. Primary B cell lymphoma of the sphenoid sinus: $\mathrm{CT}$ and MRI characteristics with correlation to perfusion and spectroscopic imaging features. Eur Arch Otorhinolaryngol 2007; 264: 1207-13.

22. Dugdale PE, Miles KA, Bunce I, et al. CT measurement of perfusion and permeability within lymphoma masses and its ability to assess grade, activity, and chemotherapeutic response. J Comput Assist Tomogr 1999; 23: 540-7.

23. Meva J, Chaudhary RK, Bhaduri D, et al. Lacunae in International Federation of Gynecology and Obstetrics (FIGO) clas- 
sification for cervical carcinoma: observational study using TNM classification as comparator. Int J Gynecol Cancer 2013; 23: 1071-7.

24. Jeong BK, Huh SJ, Choi DH, et al. Indications for endoscopy according to the revised FIGO staging for cervical cancer after MRI and CT scanning. J Gynecol Oncol 2012; 23: 80-5.

25. Guarnieri DJ, DiLeone RJ. MicroRNAs: a new class of gene regulators. Ann Med 2008; 40: 197-208.

26. Marcucci G, Mrozek K, Radmacher MD, et al. The prognostic and functional role of microRNAs in acute myeloid leukemia. Blood 2011; 117: 1121-9.

27. Murray MY, Rushworth SA, Zaitseva L, et al. Attenuation of dexamethasone-induced cell death in multiple myeloma is mediated by miR-125b expression. Cell Cycle 2013; 12: 2144-53.

28. Sun B, Zhang Y, Zhou L, et al. The proliferation of cervical cancer is promoted by miRNA-125b through the regulation of the HMGA1. OncoTargets Ther 2019; 12: 2767-76. 